Journal of Engineering Science and Technology Review 4 (3) (2011) 207 - 208 Special Issue on Econophysics

Note
JOURNAL OF

Engineering Science and

Technology Review

\title{
What is EconoPhysics?
}

www.jestr.org

\author{
P. Argyrakis*
}

Dep. of Physics, University of Thessaloniki, 54124 Thessaloniki, Greece.

\begin{abstract}
I present some ideas of how EconoPhysics has come to life in the past few years, what it deals with, the main topics of discussion in this community, and perhaps what can it offer in the future. One can see that the tools of Statistical Physics can indeed be utilized properly to understand better the behavior of the markets by constructing mathematical functions which use empirical/historical values. As the volume of such historical data explodes in recent years in an all-electronic world, it has become more and more necessary to use such new tools, thus entering a new era in the financial world, where computers play a very important role in decision making.
\end{abstract}

Keywords: EconoPhysics, probability distribution functions, correlation functions.

\section{EconoPhysics}

During the past twenty or so years [1-5] there has been an emergence of new approaches in mostly all sciences which are based on the idea that practically all entities considered, whether they are molecules, human relations, or prices of goods, exist through some sort of interaction between the multitude of their elements, which strongly affects their overall behavior. Thus, a new picture based on graph theory has spawned new ways to visualize nature, by casting these elements as network nodes and their interactions as the links (bonds) that connect these nodes. It is really amazing how in such a short period of time a huge variety of applications found their way in such pictures, and new ways were generated to visualize old problems, thus improving their understanding and/or offering explanations that were not conceivable earlier. Economics is a science which was not left out of this approach. It is a science that is trying to assign the proper value to every item that people deal with in our world. Of course, one can easily see that it is not related at all to the quantities that we commonly meet in Physics or the other Natural Sciences, i.e. the physical laws in nature. Thus, one may naively ask what is the common element between the two, how can they possibly be related, and what has prevented it all along. This is a good question. The answer is simple, it is that the values assigned to all goods of the Economy are characterized by fluctuations. But fluctuations constitute an inherent characteristic of all natural measurements. It is a subject that is studied in Statistical Physics in great detail. Thus, this common characteristic constitutes the bridge between these two disparate fields, which seemingly are concerned with so different ideas. One would then hope to apply well known

\footnotetext{
*E-mail address: panos@physics.auth.gr

ISSN: 1791-2377 @ 2011 Kavala Institute of Technology. All rights reserved.
}

methods from Statistical Physics to real-world data coming from the economy, calculate equivalent quantities that one has in Physics, but now using data such as prices of some common instrument, instead of using data arising from physical measurements. One would ask at first if this is possible, and the quick answer is that indeed it is possible, as it has been shown in a large number of cases recently.

What has additionally promoted such an approach is the huge volumes of economic data that in the past few years have been continuously generating. Of course, the reason for this is the transition to all-electronic markets, making possible to keep records of transactions at extremely small time increments, of the order of msec, something unheard of just a few years ago. This has necessitated on one hand the use of powerful computers, and on the other hand the use of powerful statistical methods in order to produce meaningful pictures of the financial markets in a very short period of time, and all this must be realized, of course, in real time. The huge increase of the volume of currency trading, and the introduction of derivatives in the 70s have all helped such trends and necessitated new approaches for the financial markets.

A first impetus to the statistical analysis of financial markets was shown by the unexpected observation in the behavior of the distribution of the price fluctuations of some instrument. It can safely be assumed as a starting point that price fluctuations occur at all scales and very importantly they are random, i.e. prices in any time increment are not correlated. This was proposed over 100 years ago as an empirical result and it was commonly accepted. If this is true one would expect intuitively to have a Gaussian or Normal distribution for the changes of the price of a stock. Recent results in the last two decades showed that this is not so. While the line shape around the maximum value of the distribution of price changes seems to be correct, the shape at the two extremes (the two tails) do not obey a Gaussian at all, but rather they agree with a Levy distribution, which 
falls much more sharply at the ends (rather than having long tails that go asymptotically to zero as in the Gaussian).

As mentioned earlier, in financial markets one is not interested only in the price itself, but also in the fluctuations of this price, which is usually called the volatility in the price. At periods of extreme volatility it is when huge market gyrations occur, such as market crashes, i.e. very sharp changes in a very short period of time, such as the one on October 1987 when the main market indices in USA fell by over $20 \%$ in one day. Conceivably, the same could happen in large upswing changes, for symmetry reasons, even though historically this is not true. Such changes belong to the tails of the distributions, which as mentioned above are far from Gaussian. Actually, if they were Gaussian, then the 1987 market crash would have a probability of $10^{-135}$ to happen, the inverse of which is larger than the size of the universe. Thus, it is now clear that the behaviors of the tails of such distributions are far from random, even though the prices themselves could be taken to be random. The interplay of these two quantities (i.e. the price of an instrument and the volatility of this price) is still a very active field of research, where no definite answers have emerged.

Such sharp changes as described above belong to the class of the so-called "rare events", which are events that happen very rarely in the world, but when they happen they have a very large impact on people. Such events could be, for example, a very large earthquake, a powerful tsunami, a catastrophic nuclear accident, or a huge swing in the financial markets discussed above. Such events may occur only once or twice within a lifetime, or let us say that they have a frequency (roughly) of the order of a century. The question then is if they can be predicted or if any information about them can be obtained ahead of time, as for example, if they have any precursors. Actually, this is the holy grail of the markets, for obvious reasons. I will give some simple arguments which I believe show that this is not possible to do. One wants to believe that we all live in a world of efficient markets. This hypothesis states that no matter how complex markets are, valuations are made in a rational way, which means that they are based on the supplydemand ratio. It also states that all players have all the available information in all parts of the world, so that if some form of arbitrage may temporarily take place, soon it will be smoothened out exactly by the same forces that have generated it and by the same mechanism. Equivalently, it is palatable to assume that if any such precursor would emerge, then the market forces would immediately take effect, making this precursor useless. Who would buy a stock if it is known that tomorrow it will be half price and the day after quarter-price? If such a real precursor would exist, then this would in effect shut down the market. For a market to operate smoothly, it is by definition required to have both buyers and sellers. When one of the two ceases to exist, then the market simply cannot operate at all. The market is not an infinite bath of financial instruments which are traded between a customer and the market. The market is simply a mechanism of matching buyers and sellers, therefore, if a catastrophic change takes place, then the balance between buyers and sellers is strongly disturbed, making trading impossible. With these thoughts, one can see that (almost by definition) such indications as predicting bubble bursts, finding precursors, etc. do not make any sense in a normal market. But just like with all other human behavior in history claims to the opposite abound and will do so in the future, because it is part of human nature.

Another property of interest is the correlation between two or more financial instruments. By this term it is simply meant how does the change in one instrument depend on the change in another. For example, if we have two stocks, how does the rise or fall of one compare to the behavior of the other in the same time period. It is well known that in daily fluctuations typically most stocks move in unison, this is true at least for the large valuation stocks, such as the ones contained in the DJ Industrials. Also, the same may be true for stocks that belong to some particular sector, such as for example, oil industry, or banking, etc. One wants to know more in detail the quantitative behavior of these stocks. Thus, it is possible to calculate the so called correlation coefficient, which answers this question. The correlation coefficient is a function similar to the correlation function, which gives, for example, the relative positions or velocities of particles in a physical system.

Finally, an area where methods from Physics can contribute is the estimation of risk and the creation of an optimal portfolio. These ideas have come to the forefront more so in recent years because of the increase of derivative trading. As we know derivatives carry a much larger risk than stocks themselves. Because they involve in addition to the valuation of the underlying instrument, the parameter of time and volatility, estimating their fair value is not an easy task. Probability theory used in physical processes can aid significantly at this point, for which there is still no general consensus. Important seminal results as the Black-Scholes theory for option pricing do not agree with empirical, real prices even today.

Summarizing, Physics can contribute to Economics by lending the mathematical tools that have been developed over the years for the study of atoms and molecules. These mainly include probability distribution functions and correlation functions. Large volumes of historical data exist today that include among others, prices of stocks, derivatives, such as options, commodities, currency pairs, index of inflation, income distribution, import-export of goods between countries, etc. to name only a few. Such functions as the ones mentioned above may give a better understanding of the mechanism that governs their behavior. It is not expected that EconoPhysics will revolutionize Economics or be able to predict trends in the Economy. But experience has shown that interdisciplinary approaches are always useful for the advancement of knowledge.

\section{References}

1. R. N. Mantegna and H. E. Stanley, "An Introduction to Econophysics: Correlations and Complexity in Finance", Cambridge University Press, (2000).

2. J. L. McCauley, "Dynamics of Markets: Econophysics and Finance", Cambridge University Press, (2004).
3. J. P. Bouchaud and M. Potters, "Theory of Financial Risks: From Statistical Physics to Risk Management", Cambridge University Press, (2000).

4. D. Sornette, "Why Stock Markets Crash: Critical Events in Complex Financial Systems", Princeton University Press, (2003).

5. B. Mandelbrot and R. L. Hudson, "The (Mis)Behavior of Markets: A Fractal View of Risk, Ruin and Reward”, Basic Books, (2004). 